\title{
Categorizing and choice reaction time performance
}

\author{
A. E. READING \\ King's College Hospital, London SE5 8AF, England \\ and \\ D. R. HEMSLEY \\ Institute of Psychiatry, London SE5 8AF, England
}

\begin{abstract}
The visual search time for the presence of both of two physical stimuli was measured under two conditions: (1) conceptual identity the targets being upper and lower case forms of the same letter (C.1); and (2) upper and lower case forms of different letters (D). It was hypothesized that C.1 would facilitate the simultaneous analysis of the features of two letters in a visual display. Each subject searched for the two classes of stimuli on alternate days. Responses in the C.1 condition were found to be significantly faster than in the $\mathrm{D}$ condition. RT was shorter for an affirmative than for a negative response, but there was no interaction between conditions and affirmative or negative responses. The results are considered in relation to the concept of categorizing.
\end{abstract}

Experiments have shown that for two-choice response tasks there may be little increase in response time whether there are two, four, or eight possible stimuli per response (Rabbitt 1959, 1964). The effect depends on the number of possible responses as well as the number of stimuli. Also, the increase in response time which is found between the conditions of one stimulus per response and two stimuli per response has been shown to disappear in a situation in which subjects are highly practiced. Thus, Broadbent and Gregory (1962) presented place names in either upper or lower case type; the subject had to make the same reaction either to HARLOW or to Harlow. Subjects were found to react as fast when the nature of the stimulus was uncertain as when all stimuli were in one size of type, the equivalence between upper and lower case letters being highly practiced. Thus with small numbers of responses, it seems that under certain circumstances man can analyze a stimulus array simultaneously for the presence either of one set of features or of another, as long as the same response is being given for both.

The aim of the present experiment is to compare the search times for the presence of both of two physically similar stimuli under two conditions: first, with the stimuli conceptually identical (C.1), upper and lower case forms of the same letter; and, second with upper and lower case forms of different letters (D). Smith (1968) has suggested that choice reaction time increases as the number of perceptually different choices increases only if the alternatives are not treated by the subject as being conceptually identical. Hence it is hypothesized that condition C. 1 will produce a faster response time than condition $\mathrm{D}$; that is, conceptual identity will

Thanks are due to J. Davidoff, Psychology Department, University College of Swansea, for his help with the running of the experiment. We also thank Dr. Leslie Henderson who sponsors this paper and takes full editorial responsibility for its content. facilitate the simultaneous analysis of the features of two letters in a visual display.

Henderson (1973) has recently reported an example of the facilitation of visual search when the targets are both cases of the same letter. However, his study utilized a letter cancellation search task involving continuous performance whereas the present investigation is of discrete choice reaction time performance with the positive response requiring the presence of both letters.

\section{METHOD}

\section{Design}

A within-subject comparison of choice reaction time under both conditions of search was employed, each subject searching for the two classes of stimuli on alternate days. Equal numbers of subjects were assigned to each of the four blocks of equivalent stimuli.

Subjects

Eight male undergraduate students volunteered to participate in the experiment.

\section{Apparatus}

The stimuli were back projected onto a screen 14 in. in front of the subject. The presentation of each slide activated a light-sensitive cell, which in turn activated a Birkbeck timer. Removal of the slide was contingent on the subject's vocal response, and this also stopped the timer.

Each slide showed a rectangular arrangement $(10 \times 8 \mathrm{~cm})$ of four letters. The angle subtended by the letters was $10 \mathrm{deg}$ $12 \mathrm{~min}$, and the angle subtended by the screen was $23 \mathrm{deg}$ $18 \mathrm{~min}$. In order to vary the position of the target information, 192 combinations of letters were used. All target letters were visually similar-for the C.1 condition, Jj, Gg, Yy, Qq; for the D condition, Jg, Gq, Qy, Yj. Each subject was assigned to a letter condition (two subjects per letter), and the order of search requirements (e.g., Jj or $\mathrm{Jg}$ first) was alternated between subjects in each of the four letter groups.

\section{Procedure}

All subjects participated in two sessions of approximately $15 \mathrm{~min}$ on consecutive days. Subjects were seated in a darkened room and informed of the exact nature of the stimuli. Before 
Table 1

\begin{tabular}{lccccc}
\hline & \multicolumn{2}{c}{ Yes } & & \multicolumn{2}{c}{ No } \\
\cline { 2 - 3 } \cline { 5 - 6 } & C.1 & D & & C.1 & D \\
\hline Mean RT (sec) & 1.03 & 1.28 & & 1.26 & 1.51 \\
SD & .521 & .584 & .795 & .665 \\
\hline
\end{tabular}

each block was presented, the subject was told the search requirements. There was a short rest period between blocks of stimuli. The intertrial interval was $8 \mathrm{sec}$, and a warning light came on $2 \mathrm{sec}$ before the presentation of each slide. Subjects were encouraged to respond as quickly as possible but without making any errors. No feedback was given during the sessions.

A baseline RT measure, consisting of 12 slides, was obtained from all subjects on both days by requiring them to search for the upper case letter of their condition. The configuration of redundant and target information for these slides remained constant across conditions. A series of 20 practice slides introduced subjects to the search requirements (C.1 or D) before the 12 test slides were presented. Within each group of slides, one half contained the target letter(s) and so called for an affirmative response. For both the practice and test slides, where the target consisted of two letters, half of the negative slides contained the upper case target letter only, the other half the lower case target letter only. The position of the target letters was varied between slides and the order of positive and negative presentations randomized.

\section{RESULTS}

The error frequency during the practice trials was $6 \%$ overall and less than $2 \%$ for the test slides. As the reaction time data was skewed, a log transform was applied. There was no significant difference in the reaction times for the baseline condition within subjects between days.

An analysis of variance compared separately the reaction times for the two conditions (C.1 and D) when the target information was present ("Yes" condition) and when it was absent ("No" condition). The means are presented in Table 1. Responses in the C.1 condition were found to be significantly faster $(p<.004)$ than in the $\mathrm{D}$ condition. There was no significant interaction between conditions and "Yes" or "No" responses $(\mathrm{p}<.821)$ although reaction time was significantly shorter $(p<.002)$ for the "Yes" response than for the "No" response.

\section{DISCUSSION}

The results are consistent with those obtained by Henderson (1973) in that search for two conceptually identical stimuli was speeded relative to search for conceptually different stimuli. Broadbent (1971), in his recent theory of information processing, has introduced the concept of "categorizing," which is relevant to the results being considered. In categorizing, the rules connecting a set of stimuli to a set of responses is changed, as when two or more combinations of features are allocated to the same category. An example suggested by Broadbent is when tall broad men and short slender men both qualify as "well proportioned," although other combinations may not. Such categorization is seen as a slowly changing process resulting from considerable practice in a particular situation. Thus C. 1 letters may be seen as allocated to the same category state through practice. Only with continued practice would one excpect the conceptually distinct letters to result in a common category state. It seems likely that with small numbers of responses man can analyze an array simultaneously for the presence of two sets of features, as long as these result in a common category state. From this, one would predict a reduction in the discrepancy between performance under C.1 and D conditions with prolonged practice.

\section{REFERENCES}

Broadbent, D. E. "Decision and stress." London and New York: Academic Press, 1971.

Broadbent, D. E., \& Gregory, M. "Human response to classes of stimuli." Nature (London), 1962, 193, 1315-1316.

Henderson, L. "Effects of letter names on visual search." Cognitive Psychology, 1973, 5, 90-96.

Rabbitt, P. M. A. "Effects of independent variations in stimulus and response probability. Nature (London), 1959, 183, 1212. Rabbitt, P. M. A. "Ignoring irrelevant information." British Journal of Psychology, 1964, 55, 403-414.

Smith, E. E. "Choice reaction time: An analysis of the major theoretical positions. Psychological Bulletin, 1968, 69, 77-110.

(Received for publication March 28, 1975.) 